\title{
Progressive Lane Analysis in the Digital Map using Fuzzy Method
}

\author{
Chia-Ming Chang and Tai-Yu Lin \\ Department of Computer Science and Engineering, Tatung University, Taipei, Taiwan, \\ E-mail: cmchang@ttu.edu.tw
}

\begin{abstract}
Since the improvement of special technology, the application of GPS becomes a low-cost and popular way. The construction of digital map is the base in many GPS applications. Because of these, the construction of the map is the most important thing in the application of GPS. GPS position tracing and satellite image analyzing were the most used methods in the construction of digital map.

In general, using GPS position trace to constructs the map, a high precision GPS system is needed, but the high precision GPS system is expensive to many people. Therefore, it cannot construct the large range of map efficiently. Using satellite image analyzing to construct the map has a big problem that the position does not match with the map when in the difference GPS coordinates datum.

In this paper, a progressive road analyzes using fuzzy technique is proposed. In this system, only the low cost GPS receivers are used. The road attribute and map are constructed only using the received data.
\end{abstract}

Keywords: Digital Map, Progressive Statistic, Fuzzy Theory, GPS, GIS.

\section{Introduction}

Nowadays, the application of GPS technology is very popular. The mobile device combining with GPS is getting more and more usual. The detail and precision of digital map are more and more necessary. So the construction of the digital map is become more important.

There are many map construction systems and the analyze methods are proposed. Bauer and Mayr proposed a system using the GPS track data and segmenting road to provide velocity attention to drivers. The new road sections needed someone to input in to the database [1]. A framework for road change detecting and database updating was proposed by Zhang and Couloigner [2], but their system needed using the image to extract the new road.
Many researches that generated map accurately and efficiently are proposed. Some papers presented that using the high-resolution satellite images to find the roads $[2,3]$. The road map obtained from satellite images may not match the position reported by GPS. Ing and Zhang analyzed the statistic GPS track data to determine the road attribute $[4,5]$, but the high precision GPS system are used.

Bruntrup proposed a map generation system based on GPS traces; the system uses the depth-first search algorithms to infer the road geometry without considering the DOP (Dilution of precision) [6].

In this paper, an automatic road attribute analyze using fuzzy technique is proposed. This system focus on the automatic road map generates using the progressive method, and uses the low cost GPS receiver to construct the road attribute.

\section{Road Analysis System}

The core of the automatic road attribute analyzes and map construct is the road data analysis, the framework of the road analysis system is illustrated in Fig. 1.

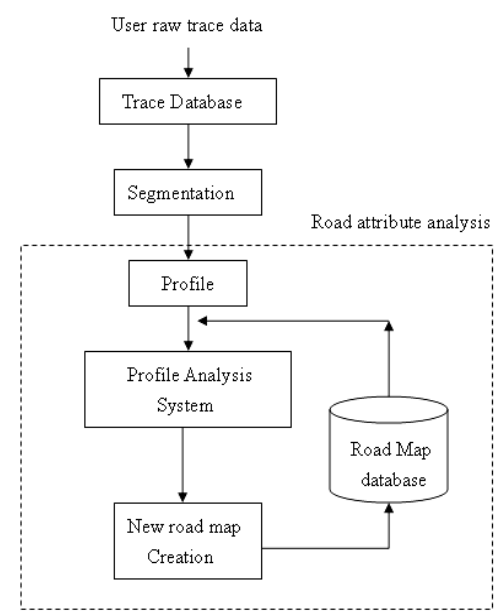

Figure 1 Flow chart of Road Analysis

The system fetches the trace data from the trace database, then segment the trace data to get many road segments shown in Fig. 2. Each road segment analysis 
is begun with the "Profile process," shown in Fig. 3, the road segment been profiled is shown.

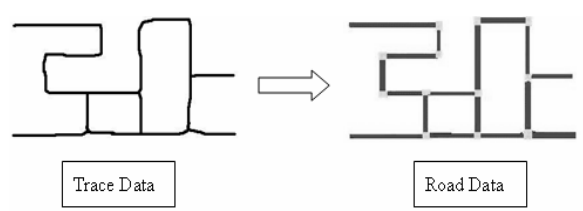

Figure 2 Segmentation

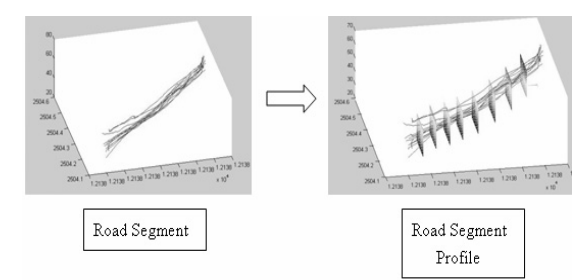

Figure 3 Profile

Profile analysis system analyzed each profile to construct the road attributes. System fetches the road map from database to analyze with the trace data, then corrects the road map stored in the database.

\section{Profile Analysis}

\subsection{Progressive Statistic Method}

Since the low-cost GPS receiver cannot provide high accuracy position information, a statistic method maybe used to reduce the position error. Considering a lot of GPS position information for a fixed point, the assumption of zero-mean position error is accepted.

The average method can be used to remove zeromean error. In this process, a lot of GPS position information is needed. Because the GPS receiver also provides the DOP information, the position information should be weighted in the average process. Therefore, the statistic position information can be obtained using

\subsection{Progressive Statistic Method}

Since the low-cost GPS receiver provides low accuracy position information, a statistic method maybe used to reduce the position error. Considering the GPS position information for a fixed point, the assumption of zero-mean position error is accepted.

The average method can be used to remove zeromean error. In this process, a lot of GPS position information is needed. Because the GPS receiver also provides the DOP information, the position information should be weighted in the average process. Therefore, the statistic position information can be obtained using

$$
\bar{x}_{k}=\frac{\sum_{i=1}^{k} x_{i} \cdot w_{i}}{\sum_{i=1}^{k} w_{i}}
$$

where $x_{i}$ is the coordinates of the $i$ th point and $w_{i}$ is weight of the $i$ th point according to DOP, respectively.

In the explanation of the profile analysis, because we have not a lot of GPS position information on the road profile to be used in the process. We use the fixed-point GPS position information to analogize the points in the profile. The fixed-point data and the best positioning point are shown in Fig. 4.

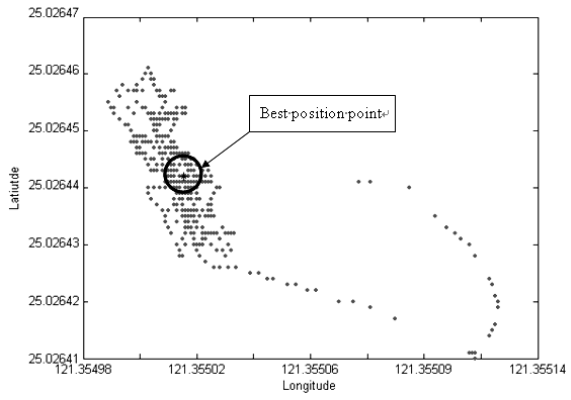

Figure 4 The best positions by statistic method

In practical situation, the number of points is not sufficient by storing all the position information of each point. Therefore, a progressive analysis method is proposed. We rewrite the weighted average equation to the progressive form as following

$$
\bar{x}_{k}=\left(\frac{\sum_{i=1}^{k-1} w_{i}}{\sum_{i=1}^{k} w_{i}}\right) \cdot \bar{x}_{k-1}+\left(\frac{1}{\sum_{i=1}^{k} w_{i}}\right) \cdot x_{k} \cdot w_{k}
$$

In the Fig. 5, the fixed-point data, the best positioning point and the progressive track are illustrated.

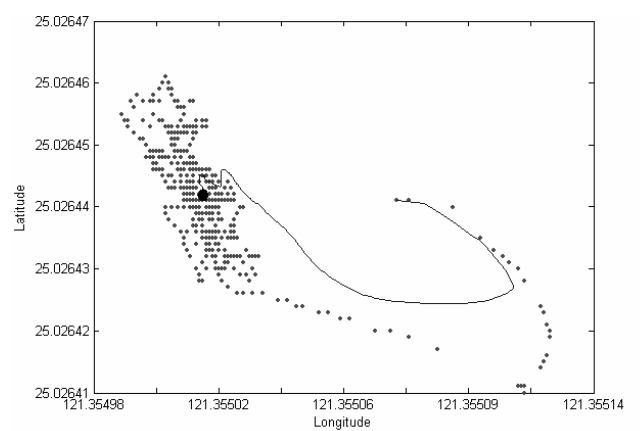

Figure 5 The moving trace of progressive form equation

The final point is same to before, it is obvious that the progressive form can also achieve to remove the zero-mean errors as the weighted averages method doing. No matter how many points are received, the best positioning point can calculated without storing all the historical data.

\subsection{Progressive Fuzzy method}

Although this progressive form of the weighted average has proved to calculate the best position successfully, some other problems in the road attribute analysis are needed to solve. Such as, "What is the 
best weight?" Therefore, a more flexible method than progressive weighted average one must be derived. The fuzzy logic theory may be used to simplify the complicated situations.

Fuzzy theory has rapidly become one of the most successful technologies for developing traditional control systems. The reason is that it is very simple to be applied without knowing much about the controlled system. Therefore, we will apply the fuzzy theory in the proposed system. Using fuzzy analysis, three parameters are considered in this system:

distance: the distance between new point and accumulative position

new_dop: the DOP of the new point

acc_dop: the DOP of the accumulative point

The results of fuzzy inference are the position correction and the new DOP. The position correction is added to the position of the accumulative point to obtain the new position of the accumulative point. The DOP is the new DOP of the accumulative point.

Two membership functions are used for deriving new distance (correction) and DOP. Each membership function has five fuzzy sets as shown in Fig. 6.
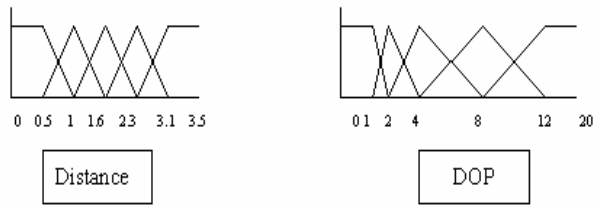

Figure 6 The membership function

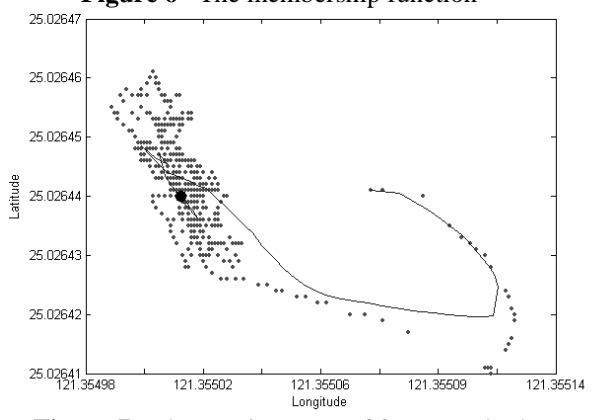

Figure 7 The moving trace of fuzzy method

The correcting value of position and the new DOP are generated when a new point is processed. Again, we use this progressive fuzzy method to analyze the fixed-point data. In Fig. 7, the moving track of the fixed-point is shown. The result obtained by applying progressive fuzzy method is very similar to the progressive statistics method.

\subsection{Multi-Lane analysis}

Using progressive fuzzy method to analyze the lane center is achieved. the number of lanes needs to be considered. To extend the progressive fuzzy method from single lane to multi-lane, the strategy of fuzzy inference should be enhanced. In the single lane situation, when encountered the position point that the distance greater than the width of one lane and the DOP is very small, the fuzzy analysis only reduce the effect to correction and DOP. However, in multi-lane situation, fuzzy analysis system will created a new lane, every position point will be used in each lane to analyze and correct the lane center.

Each point in the profile must be analyzed while it is belong to the existing lane. If the point does not belong to any existing lane, a new lane center is added. Fuzzy system analyzed all points and all lanes to update the new lane information.

\section{Implementation}

The two steps of road analysis are the segmentation and the profile analysis. The method about segmentation is to divide into two parts. First part is according to the data of course over ground; analyze the change of course to determine the join of road segment. Second part is to analysis all possible segmented point to correct the error of positional drift.

The road profiles derived from the road segment may be similar to the fixed-point data as shown in Fig. 8. The number of profiles in a road segment is corresponding to the length of the road segment.

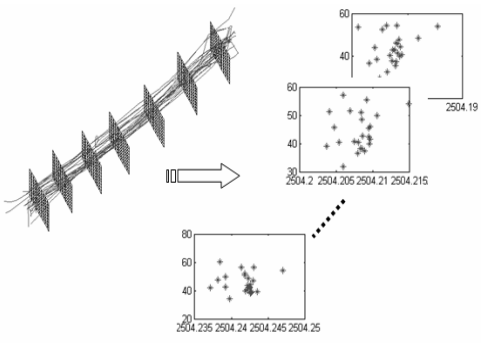

Figure 8 profile of the road segment

Two lane centers of the profiles using progressive fuzzy and statistic methods are shown in Fig. 9. It is obvious that the similar results are obtained in both methods again.

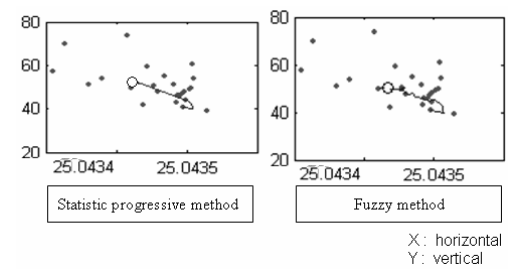

Figure 9 Profile analysis

In the multi-lane profile analysis, we use two lanes profile using fuzzy method to find out each lane center as shown in Fig. 10. From the distributing curves of profiles, there are two high peaks are found.

After finding the lane centers of all profiles, all the centers are connected to get a road segment; many 
road segments construct a road map. Figs. 11 and 12 show the final road segment about single-lane and multi-lane, respectively. System corrected each road segment in the road map when receive the user trace data, keeping the road map synchronization with the real road situation.
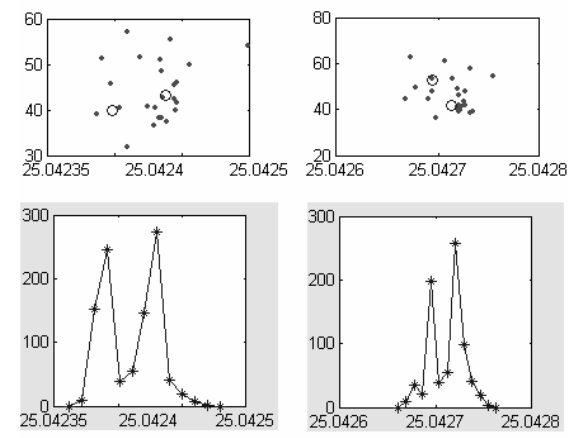

Figure 10 Multi-lane profile analyses

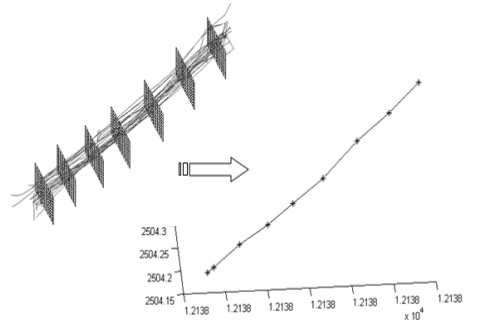

Figure 11 The final road segment of single-lane

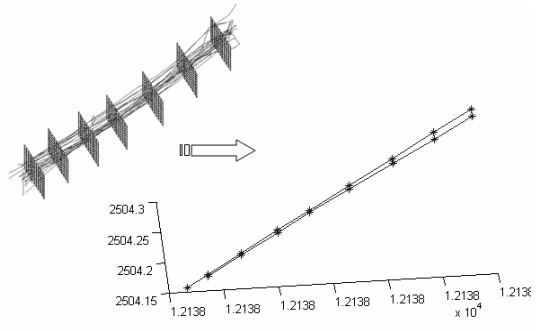

Figure 12 The final road segment of multi-lane

\section{Conclusion}

In this paper, a system that only the GPS trace data from low-cost GPS receiver are analyzed to automatic recognize the lane center to construct and update the road map without technician is proposed. The system is based on fuzzy method to realize the progressive correcting of the lane center, even the multi-lane situation. Because of the usage of progressive algorithm to generate a new value after every analysis, the huge historical data can be omitted and many storage spaces are saved. This method can also reduce plenty of calculating time when the lane center adjustment in profile analysis, because of the lane canters in each profile only the information of one point is stored, and use this point information to analyzing with the new profile point.

\section{References}

[1] O. Bauer and R. Mayr, "Road Database Design for Velocity Profile Planning," Proc. of IEEE Conf. on Control Applications, vol. 2, pp. 13561361, 2003.

[2] Q. Zhang and I. Couloigner, "A framework for road change detection and map updating," The Intl. Archives of the Photogrammetry, Remote Sensing and Spatial Information Sciences, Vol. 34, 2004.

[3] W. Shi and C. Zhu, "The Line Segment Match Method for Extracting Road Network From High-Resolution Satellite Images," IEEE Trans. on Geoscience and Remote Sensing, VOL. 40, NO. 2, pp. 511-514, 2002.

[4] I. H.-J. Euler and C. D. Hill, "Real-time Precise GPS for Railroad Mapping," IEEE Position Location and Navigation Symposium, pp. 437443, 1996.

[5] W. Zhang and V. Taliwal, "Using Lane Tracker Data to Improve Lane-level Digital- Maps Created With Probe Vehicle Data," IEEE Trans. on Intelligent Systems, vol.1 pp. 585-589, 2003.

[6] R. Bruntrup, S. Edelkamp, and S. Jabbar, B. Scholz, "Incremental Map Generation with GPS Traces," Proc. of the 8th Intl. IEEE Conf. on Intelligent Transportation Systems, Vienna, Austria, pp. 574-579, Sep. 13-16, 2005

[7] C.-J. Lin, Y.-Y. Chen, and F.-R. Chang, "Fuzzy Processing on GPS Data to Improve the Position Accuracy," Fuzzy Systems Symposium on Soft Computing in Intelligent Systems and Information Processing, pp. 557-562, 1996.

[8] M. Kurihara, H. Nonaka and T. Yoshikawa, "Use of Highly Accurate GPS in Network-Based Barrier-Free Street Map Creation System," Proc. of IEEE Conf. on Systems, Man and Cybernetics, vol. 2, pp. 1169-1173, 2004.

[9] W. Holzapfel, "Road Profile Recognition for Autonomous Car Navigation and Navstar GPS Support," IEEE Trans. on Aerospace and Electronic Systems, Vol. 39, pp. 2-12, 2003.

[10] J. Fawcett and P. Robinson, "Adaptive Routing for Road Traffic," Proc. IEEE Computer Graphics and Applications, Vol. 20, pp.46-53, May-June, 2000.

[11] S. Rogers, P. Langley, and C. Wilson, "Mining GPS Data to Augment Road Models," Proc. of the 5th ACM SIGKDD Intl. Conf. on Knowledge Discovery and Data Mining, pp. 104-113, 1999.

[12] S. Rogers, "Creating and Evaluating Highly Accurate Maps with Probe Vehicles," Proc. of IEEE Conf. on Intelligent Transportation Systems, pp. 125-130, 1-3 Oct. 2000. 